\title{
AN INEQUALITY FOR A POSITIVE REAL FUNCTION
}

\author{
JEONG SHEOK UME
}

\begin{abstract}
In this paper, using a suitable mapping, we show that the result of $\mathrm{H}$. Alzer can be extended and open problem is proposed.
\end{abstract}

Mathematics subject classification (2000): 26D20.

Key words and phrases: inequality, strictly increasing.

\section{REFERENCES}

[1] H. Alzer, On an Inequality of H. Minc and L. Sathre, J. Math. Anal. Appl. 179 (1993), 396-402.

[2] N. ElezoviĆ AND J. PeCARIĆ, On Alzer's inequality, J. Math. Anal. Appl. 223 (1998), 366-369.

[3] J. S. Martins, Arithmetic and geometric means an applications to Lorentz sequence spaces, Math. Nachr. 139 (1988), 281-288.

[4] H. Minc AND L. SATHRE, Some inequalities involving ( $n !)^{\frac{1}{r}}$, Proc. Edinburgh Math. Soc. 14 (1964-1965), 41-46.

[5] J. SÁNDOR, On an inequality of Alzer, J. Math. Anal. Appl. 192 (1995), 1034-1035.

[6] J. S. UME, An elementary proof of H. Alzer's inequality, Math. Japonica 44 (1996), 521-522. 